\title{
Imagerie et chirurgie guidée
}

\author{
Fortin T \\ Département de Chirurgie buccale, Faculté d'Odontologie, Lyon, France \\ thomas.fortin@univ-lyon1.fr
}

La chirurgie assistée par ordinateur est une véritable aide ou complément aux traitements implantaires conventionnels. Dans son principe, elle nécessite le recours à une imagerie tridimensionnelle. D'une manière générale, les systèmes permettant les Gestes MédicoChirurgicaux Assistés par Ordinateur (GMCAO) sont composés d'un logiciel de planification du geste opératoire et d'un outil technologique pour transférer ce geste sur le site opératoire. Ils peuvent être divisés en trois catégories selon l'autonomie qui est laissée au chirurgien : passifs, semi-actifs et actifs. Avec les systèmes passifs, l'assistance au geste opératoire consiste à rendre possible la comparaison entre le geste planifié avant l'intervention et celui que le chirurgien réalise sur le site opératoire, en temps réel. Les technologies de navigation, assimilables au "Guidage Par Satellite ", sont les principaux types de systèmes passifs proposés pour les GMCAO. Le praticien visualise en direct la progression de son instrument dans l'image préopératoire. Les avantages principaux des systèmes passifs sont la flexibilité et la sécurité. Avec les systèmes semi-actifs, le geste chirurgical est contraint physiquement à suivre l'axe prédéfini.

Les avantages de cette technique découlent de la prédictibilité du résultat à la planification. Ainsi La chirurgie guidée apporte plus de sécurité dans l'évitement des obstacles anatomiques et permet la préparation avant l'intervention d'une prothèse adaptée à la situation virtuelle des implants et donc une mise en charge immédiate de ces implants. Cette procédure chirurgicale modifiée apporte un confort accru au patient : réduction de la durée d'intervention et réduction des suites postopératoires (douleurs, œdème) en raison de l'absence de lambeau et de manipulation périostée, même dans des cas d'implantations multiples chez un édenté complet ou subcomplet. Il en résulte une possible limitation des remodelages osseux péri-implantaire dans le cadre des interventions chirurgicales transmuqueuses bien qu'il n'y ai pas de preuve formelle en ce sens à ce jour.

La chirurgie implantaire assistée par ordinateur est une procédure thérapeutique complémentaire qui peut permettre de limiter l'invasivité des techniques conventionnelles. Elle nécessite une imagerie en trois dimensions qui doit la faire réserver à des situations cliniques justifiant son utilisation. La qualité des résultats est liée à celle de la réalisation de l'ensemble des étapes et nécessite une courbe d'apprentissage équivalente à celle des procédures conventionnelles. La validation des indications et la prédictibilité des résultats doit s'appuyer sur la poursuite d'études cliniques spécifiques. 